EPJ Web of Conferences 41, 10015 (2013)

DOI: $10.1051 /$ epjconf/20134110015

(C) Owned by the authors, published by EDP Sciences, 2013

\title{
Fully coherent spectral broadening of femtosecond pulses from an Er:fiber system
}

\author{
S. Kumkar, G. Krauss, D. Brida, and A. Leitenstorfer \\ Department of Physics and Center for Applied Photonics, University of Konstanz, D-78457 \\ Konstanz, Germany
}

\begin{abstract}
Coherence properties of the ultrabroadband output from a highly nonlinear germanosilicate fiber pumped by a femtosecond Er:fiber source are investigated. Conditions necessary to achieve full spectral coherence are demonstrated experimentally and analyzed theoretically.
\end{abstract}

\section{Introduction}

Due to their compactness and stability, mode locked fiber lasers are gaining attractivity for ultrafast applications. A key feature is spectral broadening in highly nonlinear fibers that allows wide tunability and ultrashort pulse generation at extremely high stability and low intensity noise.

Owing to their tailorable dispersion profile, micro structured fibers (PCF) are commonly used for this task [1]. But these devices are known as a possible cause of incoherence. The reason are modulation instabilities (MI) which represent exponentially growing solutions of the nonlinear Schrödinger equation (NLSE) for intensity fluctuations [2]. This effect randomly influences the soliton fission process which is widely exploited for supercontinuum generation [3], leading to a degradation of coherence properties of the generated spectrum [1]. Nevertheless, certain conditions have been proposed to allow generation of fully coherent spectra [4].

Here we present a broadband laser source based on Er:fiber technology that fulfills these conditions by exploiting short pieces of highly nonlinear germanosilicate bulk fibers (HNF) [5]. Coherence of the generated spectra is qualitatively predicted by a theoretical model and verified experimentally. This strategy allows generation of tunable ultrashort pulses with a clean pulse profile [6] and the seeding of subsequent amplification stages in the Tm and $\mathrm{Yb}$ gain windows [5].

\section{Generation of tailor-cut spectra}

In order to efficiently drive nonlinearities we employ an Er:fiber oscillator-amplifier system providing $8 \mathrm{~nJ}$ pulses with a duration of $120 \mathrm{fs}$. The supercontinuum generation takes place in an assembly of two different fibers, a standard telecom fiber followed by a highly nonlinear germanosilicate bulk fiber. The first fiber with a length of $9 \mathrm{~cm}$ serves as a precompression element by exploiting soliton formation processes. Pulse durations as low as $20 \mathrm{fs}$ are achieved before coupling to the directly spliced HNF with a length of between a few millimeters and $1 \mathrm{~cm}$. Here the pulses split up into a dispersive part travelling under the influence of normal dispersion and a solitonic component in the anomalous dispersion regime. Subsequently, the broadening process is dominated by spectral repulsion caused by four-wave mixing processes of the two pulses

This is an Open Access article distributed under the terms of the Creative Commons Attribution License 2.0, which permits unrestricted use, distribution, and reproduction in any medium, provided the original work is properly cited. 
propagating with similar group velocities. Simulations based on the NLSE allow us to design tailorcut fiber assemblies. In combination with the freedom of varying the chirp of the input pulse via a prism compressor in front of the HNF assembly, we are able to generate tunable spectra in the wavelength range spanning from $800 \mathrm{~nm}$ to $2200 \mathrm{~nm}$. This technology allows us to synthesize ultrashort pulses approaching the single-cycle limit [7].

\section{Coherence properties}

As in most cases modulation instabilities and their influence on the soliton fission process are the main reason for incoherence, we first have a look at the characteristic length scales on which these effects arise. The soliton fission length is given by $\mathrm{L}_{\text {fiss }}=T^{2}\left(\beta_{2} N\right)^{-1}$ and the onset length for MI, $L_{M I}$, can be estimated to be $\mathrm{L}_{\mathrm{MI}}=16\left(\mathrm{P}_{0} \gamma\right)^{-1}$. T is the pulse duration, $\mathrm{P}_{0}$ the peak power and $\mathrm{N}$ the corresponding soliton order [1]. $\beta_{2}$ represents the group velocity dispersion of the fiber and $\gamma$ the nonlinearity parameter. These values and characteristic scales are listed in Table 1 for typical fibers and pulse parameters.

Table 1. Typical parameters of pump pulses and nonlinear fibers together with resulting characteristic length scales for soliton fission and the occurrence of modulation instabilities.

\begin{tabular}{|c|c|c|c|c|c|c|c|}
\hline Fiber & $\boldsymbol{\beta}_{\mathbf{2}}\left[\mathbf{p s}^{\mathbf{2}} \mathbf{/ k m}\right]$ & $\boldsymbol{\gamma}[\mathbf{( W \cdot k m})^{-\mathbf{1}}$ & $\mathbf{T}[\mathbf{f s}]$ & $\mathbf{P}_{\mathbf{0}}[\mathbf{k W}]$ & $\mathbf{N}$ & $\mathbf{L}_{\text {fiss }}[\mathbf{m m}]$ & $\mathbf{L}_{\mathbf{M I}}[\mathbf{m m}]$ \\
\hline PM-SMF & -19.7 & 1.3 & 125 & 25 & 4.6 & 159 & 496 \\
\hline HNF & -4.41 & 9 & 20 & 200 & 12.7 & 7.1 & 9 \\
\hline
\end{tabular}

In order to experimentally examine the coherence properties of our system, we employ an interferometer consisting of two independent Er:fiber amplifier branches which are seeded by the same oscillator. After each amplifier, we implement identical highly nonlinear fiber assemblies consisting of $9 \mathrm{~cm}$ standard fiber and $8 \mathrm{~mm}$ of HNF to generate a broadband soliton and dispersive wave, respectively [see green and red dashed lines in Fig. 1 (a) and (b)].

The two beams are collimated and subsequently overlapped at a 50/50 beam splitter. The temporal delay between the pulses may be adjusted via a variable delay line and the relative intensity can be changed in one branch via a variable neutral density filter. Perfect spatial overlap is ensured by coupling the collinear beams into a polarization-maintaining monomode fiber. A polarizer is used to project the polarization of both parts into the same direction. The intensity is kept low enough to prevent nonlinear propagation in this fiber. A slight temporal delay of the pulses with respect to each other leads to a spectral modulation, with a period determined by the offset. The modulation depth of the interference fringes is a direct measure for the first-order coherence of the spectra. The dispersive part is analyzed via an optical spectrum analyzer with a resolution set to $0.06 \mathrm{~nm}$ [Fig. 1(a)]. To our pleasant surprise, we find a modulation depth higher than $99.5 \%$ in the entire region where the amplitude of both spectra can be perfectly equalized. The missing $0.5 \%$ are due to drift between the 5-m-long arms of the free-running interferometer.

For the characterization of the solitonic part we use an extended InGaAs spectrometer. Here we find a modulation depth better than $98 \%$ throughout the entire spectrum [Fig. 1(b)]. This value is limited by the spectral resolution of $2 \mathrm{~nm}$ achieved in this measurement. 

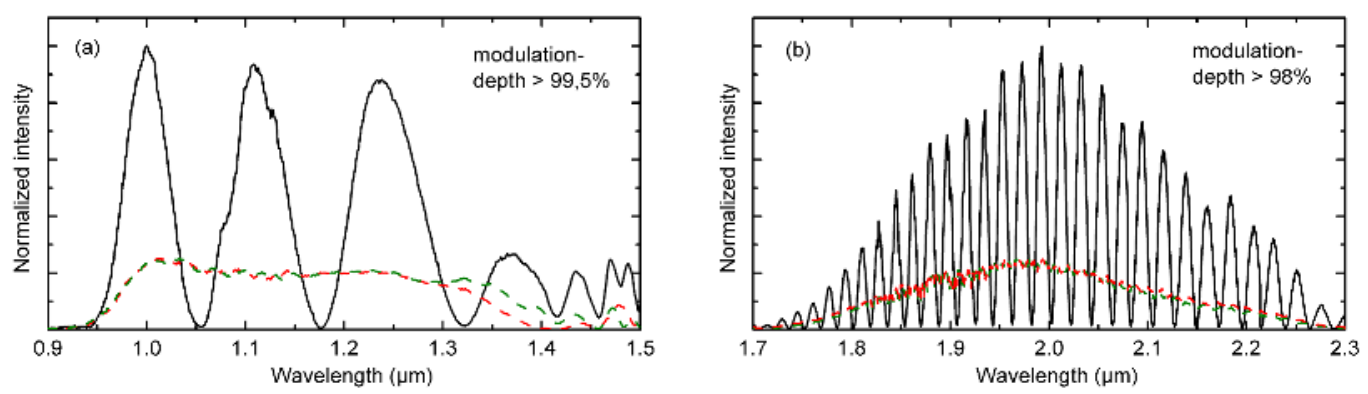

Fig. 1. Ultrabroadband emission spectra generated in the two separated combinations of Er:fiber amplifier and highly nonlinear fiber (dashed lines) and the interference of both branches after superimposition with small temporal delay (black lines).

We conclude that our approach to generate highly flexible ultrabroad and tuneable spectra is capable to maintain full coherence throughout the entire supercontinuum, even in its spectral wings. An attractive application of such pulses is the coherent seeding of high-power amplifier systems based on $\mathrm{Yb}$ : and Tm:fibers. Especially in the case of Thulium amplifiers, there exists a lack of ultrabroadband and coherent seed sources which can be overcome with this approach [5]. In combination with the capabilities for single-cycle pulse synthesis [7] and passive phaselocking [8], this technology opens up opportunities for extreme nonlinear optics at high repetition rates and with unprecedented stability.

\section{References}

[1] J. M. Dudley, G. Genty, and S. Coen, Rev. Mod. Phys. 78, 1135 (2006)

[2] K. Tai, A. Hasegawa, and A. Tomita, Phys. Rev. Lett. 56, 135 (1986).

[3] A. Husakou and J. Herrmann, J. Opt. Soc. Am. B 19, 2171 (2002).

[4] G. A. Nowak, J. Kim, and M. N. Islam, Appl. Opt. 38, 7364 (1999).

[5] S. Kumkar, G. Krauss, M. Wunram, D. Fehrenbacher, U. Demirbas, D. Brida, and A. Leitenstorfer, Opt. Lett. 37, 554 (2012).

[6] A. Sell, G. Krauss, R. Scheu, R. Huber, and A. Leitenstorfer, Opt. Express 17, 1070 (2009).

[7] G. Krauss, S. Lohss, T. Hanke, A. Sell, S. Eggert, R. Huber, and A. Leitenstorfer,

Nature Photon. 4, 33 (2010).

[8] G. Krauss, D. Fehrenbacher, D. Brida, C. Riek, A. Sell, R. Huber, and A. Leitenstorfer, Opt. Lett. 36, 540 (2011). 